\title{
El dictamen pericial odorológico: Breve análisis de su efectividad en la provincia de Guantánamo*
}

\section{The Odorological Expert Opinion: Brief Analysis of its Effectiveness in the Province of Guantánamo}

Msc Jorge Aquiles Fuentes Estévez** https://orcid.org/0000-0002-7698-7257

Msc Vladimir Naranjo Gómez ${ }^{* * *}$ https://orcid.org/0000-0002-8315-2872

Msc Guido Fabián González Sosa*** https://orcid.org/0000-0002-3012-4472

http://dx.doi.org/10.21503/lex.v18i25.2098

*El artículo es resultado de la actividad investigativa de sus autores que corresponde a la línea de investigación del Departamento de Derecho de la Universidad de Guantánamo, referida al Derecho Penal y Criminológico y sus retos en el siglo XXI.

**Licenciado en Derecho, magister en derecho Penal. Profesor de Criminología y

Criminalística del Departamento de Derecho de la Universidad de Guantánamo, Cuba. Correo electrónico: jorgeaquiles@cug.co.cu

*** Licenciado en Derecho, magister en Dirección. Profesor asistente de Criminología, Departamento de Derecho, Universidad de Guantánamo, Cuba.

Correo electrónico: vladimirng@cug.co.cu

****Licenciado en Derecho, magister en Derecho Penal. Profesor de Derecho Penal de la Universidad de Guantánamo, Cuba.

Correo electrónico: guidogs@cug.co.cu

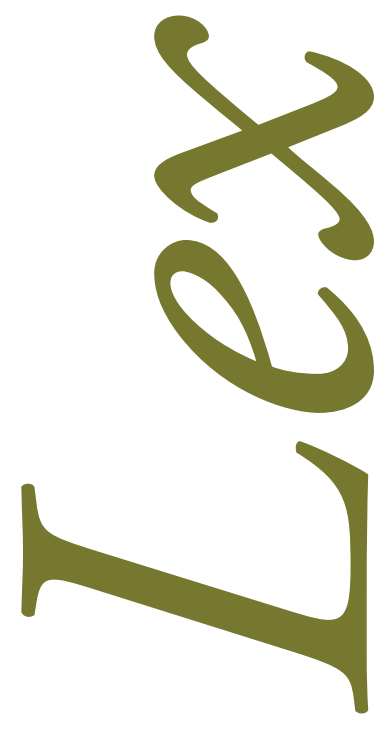
(c) (c) Los autores. Artículo publicado por la Revista Lex de la Facultad de Derecho y Ciencias Políticas de la Universidad Alas Comercial-Compartir Igual 4.0 Internacional.(http://creativecommons.org/licenses/by-nc-sa/4.0/), que permite el uso no comercial, distribución y reproducción en cualquier medio, siempre que la obra original sea debidamente citada. 


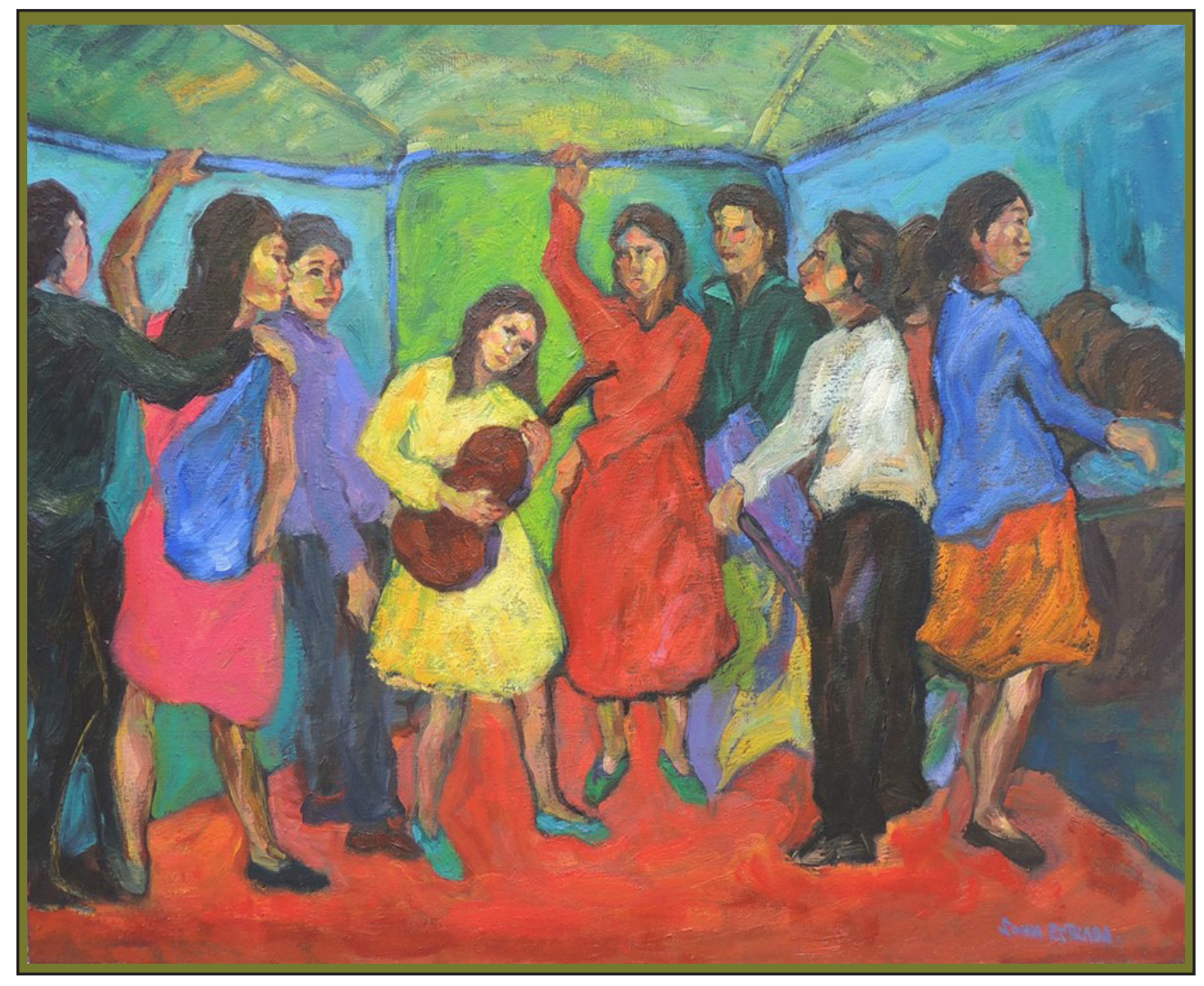

Artista de la calle. Óleo sobre lienzo $65 \times 81 \mathrm{~cm}$. Sonia Estrada Melgarejo (pintora peruana, Ancash) 


\section{RESUMEN}

La presente investigación pretende fundamentar desde una perspectiva histórica, doctrinal, legal el estudio de la odorología criminalística como técnica, a partir de su análisis jurisprudencial en la academia patria, referido a su aplicación y evaluación del dictamen pericial odorológico como medio de prueba en el proceso penal cubano, partiendo de la experiencia de su empleo en la provincia de Guantánamo. Para el logro de los resultados obtenidos se utilizaron los siguientes métodos generales de las ciencias: análisis síntesis, inducción-deducción, teórico jurídico, jurídico comparativo y exegético, así como las técnicas de investigación, la entrevista, el análisis de documentos, el análisis secundario, el estudio de casos y el análisis estadístico.

Palabras claves: dictamen pericial, huella olorosa, odorología criminalistica, impresión olorosa.

\section{ABSTRACT}

The present investigation tries to base from a historical, doctrinal, comparative and legal perspective the study of the criminalistic odorology as a technique, from its jurisprudential analysis in the homeland academy, referred to its application and evaluation of the Odorological expert opinion as a means of evidence in the Cuban criminal process, based on the experience of his employment in the province of Guantánamo. For the achievement of the results obtained the following general methods of the theoretical sciences, synthesis analysis, induction-deduction and specific methods theoretical, legal, comparative and exegetical theoretical guidance were used, as well as research techniques, interviews, analysis of documents, secondary analysis and case studies.

Keywords: expert opinion, odorous footprint, criminalistic, odorology, odorous impression. 


\section{INTRODUCCIÓN}

La Odorología Criminalística tiene un amplio empleo en la investigación y esclarecimiento de los hechos delictivos por los órganos de enfrentamientoy de Justicia que intervienen (Fiscalía, Bufete y Tribunales), de todo el país, la acusación de los autores y la imposición de sanciones penales.

El proceso de obtención y procesamiento de la huella olorosa es bastante sencillo, siempre que el mismo se realice cumpliendo estrictamente con las normas y procedimientos creados al respecto.

Esta técnica criminalística se ha tornado en cierta medida polémica por la duda que ha generado sobre sus reales resultados y lo veraz de sus resultados en la determinación de una o varias personas que hayan cometido un hecho delictivo, de esto se han desprendido diversos criterios en el orden jurídico. No obstante, su uso más frecuente, utilizando métodos y medios para su aplicación y sus resultados han permitido que la Criminalística acepte esta como una de sus técnicas.

La Odorología Criminalística surge en la década de los sesenta del siglo XX, en la antigua Unión Soviética, donde se realizaron los primeros experimentos con olores en conserva, estas investigaciones se desecharon a finales de esta década, pero la República Democrática Alemana retomó estos trabajos y desarrolló la especialidad, exponiendo sus avances en el Simposio Internacional de Criminalística, celebrado en ese país en el año 1972. En Cuba, en el año 1989 se decide comenzar la construcción del Laboratorio de Odorología, con la experiencia de algunos compañeros que habían visitado estos países antes mencionados y con la ayuda además de material bibliográfico ruso que abordaba el tema.

En 1991 se desarrollan las primeras pruebas de campo en la Novena Unidad de la Policía Nacional Revolucionaria de Arroyo Naranjo en La Habana, obteniéndose los primeros resultados y decidiéndose extender la técnica a la capital del país y posteriormente en 1993, al resto del territorio nacional. Además de Cuba, en el mundo emplean la Odorología como técnica criminalística: Rusia, Dinamarca, Holanda, Bélgica, Suecia, Alemania, Hungría, España, Colombia, Venezuela y México entre otros, teniendo cada país un diferente nivel de desarrollo, pero todos emplean un método similar en su aplicación. 
La literatura estudiada confirma que hay consenso en definir la especialidad de odorología criminalística como la que "se encarga del estudio del mecanismo de formación de la huella olorosa, así como el de los medios y métodos empleados para su captación, conservación y posterior comparación con las impresiones olorosas de los ciudadanos sospechosos de un delito investigado" ${ }^{1}$.

Mediante esta especialidad de la criminalística se cumple de forma rigurosa con las fases de recolección de las huellas y evidencias; desde el mecanismo de formación para poder proceder a su búsqueda en el lugar de los hechos, hasta el desarrollo de medios y métodos que permitan su revelación, fijación, extracción y conservación para su posterior comparación.

Para la odorología criminalística es esencial el empleo del método particular de comparación, porque el resultado final de la investigación de la huella de olor se sustenta en comparar las huellas extraídas del lugar del hecho con las muestras de olor que se les toma a los sospechosos del supuesto hecho delictivo que se está investigando.

En el análisis del dictamen pericial de la huella de olor pesan dos grandes problemas, el primero de ellos referidos a la resistencia que ha tenido en el orden histórico darle el valor que justamente tiene la prueba indiciaria en la investigación criminal, aún en la mente humana está vigente la duda acerca de su papel en la determinación de la verdad; y en segundo lugar por ser una prueba de reciente descubrimiento que surge del aporte de los nuevos descubrimientos científicos al esclarecimiento del hecho delictivo; con independencia del auge que ha alcanzado su aplicación en los últimos años.

Los medios y métodos utilizados en la aplicación de la técnica, así como los resultados obtenidos en la misma posibilitan que la huella de olor se convierta en indicio probatorio en el proceso penal en Cuba. Tabla con la información brindada por el Laboratorio Provincial de Criminalística de Guantánamo, relacionada con las huellas levantadas desde el 2015 hasta el mes de septiembre de 2019 y el comportamiento de la investigación de las huellas de olor.

\section{VALORACIÓN ESTADÍSTICA DE LA EFECTIVIDAD DE LA HUELLA DE OLOR EN LA PROVINCIA DE GUANTÁNAMO}

Para alcanzar los objetivos que pretendemos en esta investigación, no apoyamos en los datos acumulados acerca de las huellas levantadas por el Laboratorio Provincial de Criminalística de la provincia de Guantánamo, y que reflejamos en la tabla que seguidamente exponemos:

1. Colectivo de autores: Temas de Criminalística, (La Habana: Editorial Universitaria Félix Varela, 2007),41. 


\begin{tabular}{|c|c|c|c|c|c|}
\hline Años & 2015 & 2016 & 2017 & 2018 & $\begin{array}{c}2019 \\
\text { (nonestre) }\end{array}$ \\
\hline Total de huellas levantadas & 3536 & 3801 & 3669 & 2885 & 2657 \\
\hline De ellas fueron huellas de olor & 1152 & 1244 & 1514 & 1056 & 744 \\
\hline \% con respecto al total de huellas & 32.5 & 32.7 & 41.2 & 36.6 & 28 \\
\hline Cuantas huellas de olor resultaron & 362 & 382 & 672 & 559 & 347 \\
\hline positivas & $31,4 \%$ & $30,7 \%$ & $44,3 \%$ & $52,9 \%$ & $46,6 \%$ \\
\hline
\end{tabular}

Al evaluar los datos estadísticos sobre las huellas procesadas en el período comprendido desde el año 2015 hasta el mes de septiembre del 2019, por el Laboratorio Provincial de Criminalística de la provincia de Guantánamo, arribamos a las siguientes consideraciones:

a) En el período analizado como promedio el $34.2 \%$ del total de huellas que se levantan en el lugar de los hechos son huellas de olor; lo cual indica, sin discusión alguna, el aporte de su análisis en el esclarecimiento del hecho investigado, debido a la frecuencia con que es detectada en el lugar de los hechos.

b) A lo anteriormente señalado se une el hecho de que, como promedio, el $41.1 \%$ de las huellas de olor levantadas tuvieron un valor positivo, aspecto de una gran relevancia para la investigación y esclarecimiento del hecho delictivo que se esté investigando.

Se demostró que no solamente es alta la frecuencia en que se levantada huellas de olor en el lugar de los hechos, sino que alcanza una gran efectividad su valor como prueba indiciaria.

c) En los dos últimos años del período investigado, se ha elevado la efectividad de la huella de olor. En el año 2018 el porciento de huellas positiva alcanzó el 52.9 y hasta el mes de septiembre del año 2019 el porciento fue de 46.6. No obstante resulta insuficiente para los encargados del procesamiento e investigación del delito la utilización de las huellas de olor con valor identificativo en la comparación con las muestras de sospechosos y acusados en los procesos penales, aspecto que requiere especial atención.

A modo de ilustración mostramos en un gráfico de barra el comportamiento de la investigación de la huella olorosa en la provincia de Guantánamo en el período estudiado: 


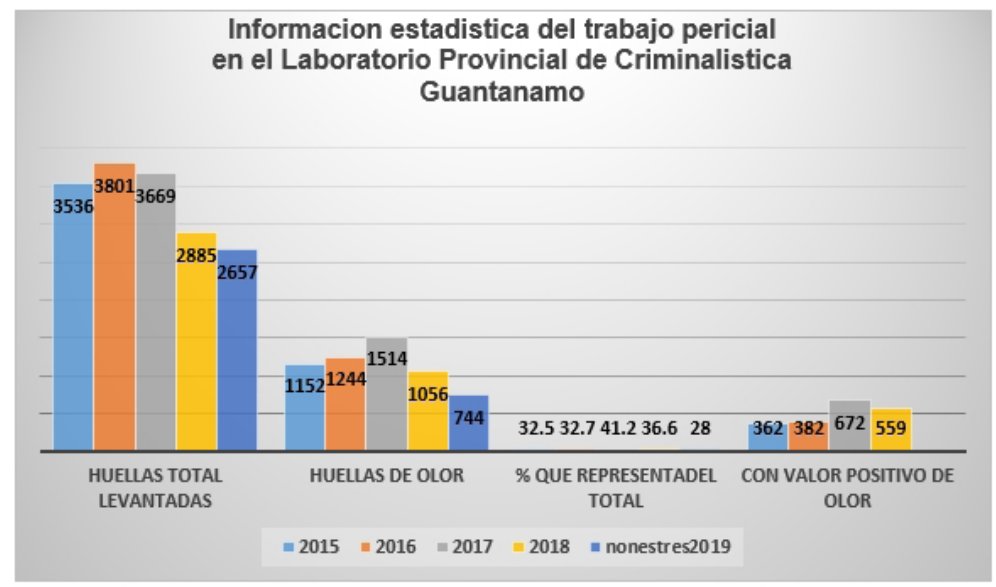

Otro aspecto a considerar en esta investigación es la alta efectividad que tiene la búsqueda, revelación, fijación, extracción, conservación e investigación de la huella de olor en la provincia de Guantánamo. En primer lugar, en la fase de búsqueda se denota una gran certeza, dada la cantidad de huellas que se localizan, como paso elemental para poder proceder al cumplimiento del resto de las fases de recolección de las huellas y evidencias. Se aprecia además una alta precisión en la fase de extracción y conservación.

Es preciso acotar la necesaria relación que tiene que existir entre el acta de inspección del lugar del hecho y la huella de olor investigada. En la primera diligencia de instrucción mencionada tiene que anotarse con exactitud el lugar exacto donde fue localizada y levantada la huella de olor. Con este proceder metodológico se fortalecer el estrecho vínculo que existe entre ambas pruebas. Suma importancia reviste cumplir en el orden metodológico con la calidad en la obtención tanto de la huella olorosa como de la impresión de sospechosos, así como la correcta guarda y custodia de la misma, que en ocasiones el incumplimiento de esta genera que no sea apreciada por el Tribunal en su momento.

\section{RESULTADO DE LAS VALORACIONES DEL ESTUDIO DE CASOS}

Para ilustrar acerca del aporte de la búsqueda, revelación, fijación, extracción, conservación y análisis de la huella olorosa, estudiamos un total de nueve casos donde en el proceso investigativo y en la formulación de las conclusiones provisionales, el fiscal sostiene las conclusiones finales de la investigación y la acusación, en el peritaje odorológico; y en su caso del órgano juzgador fundamenta su sentencia en el análisis ponderado de los resultados del informe pericial de huella de olor.

La huella olorosa también se constituye en parte del total de las pruebas indiciarias, dirigidas a producir elementos probatorios que contribuyan a demostrar la ocurrencia de un hecho delictivo y "desmontar" la presunción de inocencia, como requisito previo a formar en el investigador, en el fiscal y en el tribunal juzgador la certeza de culpabilidad. 
Es elemental partir de la convicción del investigado y del fiscal, de la importancia que tiene para el esclarecimiento del presente hecho delictivo, la realización del dictamen pericial de odorología.

La existencia de indicios o elementos probatorios es el referente para lograr la certeza de culpabilidad en los jueces, por lo que resulta necesarioel respaldo que le imprime al dictamen pericial la exposición científica del perito en el juicio oral, por lo que reproducir al dictamen pericial en el juicio oral sin la presencia del perito no es suficiente en el esclarecimiento completo, multilateral y objetivo del hecho delictivo.

Los casos estudiados se corresponden generalmente con delitos que afectan la propiedad, aunque no es solamente en esta tipología delictiva sobre las que recae la fuerza probatoria de este tipo de peritaje. En casos, como los delitos vinculados al uso ilegal de drogas y el de salida ilegal del territorio nacional, también ha contribuido al esclarecimiento multilateral, completo y objetivo del hecho, la aplicación de la técnica criminalística de odorología.

Se puede afirmar categóricamente que los tribunales cubanos le conceden igual importancia al peritaje de odorología, que a cualquiera de las pruebas que conforman el pliego acusatorio de la causa en cuestión.

En todos los casos estudiados la huella de olor contribuyó a precisar un elemento que a todas luces resulta vital, ubicar al acusado en el lugar de los hechos; demostrar que irrefutablemente el acusado estuvo presente en el lugar donde se cometió el hecho delictivo; que a pesar de que no es una prueba determinante de que el presunto autor del delito fue el definitiva el que lo cometió, porque esta certeza parte de la valoración integral de todas las pruebas e indicios; si tiene un peso importante en la determinación de la culpabilidad.

En este aspecto es preciso acotar que de manera se comporta la búsqueda de las impresiones olorosas de posibles sospechosos para el cotejo de las huellas olorosas levantadas en el lugar del suceso, ya que consta en los expedientes investigativos y de fase preparatoria un número importante de huellas sin que se soliciten peritajes odorológicos que confirmen o no quién o quiénes las produjeron, lo que se traduce en una pérdida de recurso de todo tipo, incluyendo el tiempo dedicado al trabajo en el lugar de los hechos.

Otro aspecto importante es confirmar en el proceso de interrogatorios de sospechosos (acusados), la ubicación de la huella olorosa levantada en el lugar de los hechos, lo que posibilitaría tanto al investigador, fiscal y jueces apreciar este elemento también en momento procesal que corresponde a cada uno de ellos, en la formación de la convicción necesaria para la emisión de su conclusión o fallo.

Se confirmó además que es escasa en el proceso de instrucción la participación del perito, que concurre al lugar de los hechos y del que procesa en el Laboratorio de Criminalística el peritaje, como apoyo al interrogador, o sea, para contribuir, en el orden operativo y psicológico, a la confesión del acusado y en consecuencia al esclarecimiento del hecho. 
La valoración de las sentencias estudiadas confirma además de no solamente tiene transcendencia levantar la huella de olor en el lugar del hecho y comparar con la muestra de olor tomada a los presuntos autores, sino además que es de suma importante valorar el sitio exacto donde fue ubicada y fijada la huella de olor en el lugar de los hechos, por el valor probatorio que siempre tiene este elemento en la certeza de culpabilidad que debe formarse en los jueces. Para ilustrar al respecto nos apoyamos en la siguiente valoración que el Tribunal Popular Provincial de Guantánamo realizó, "asimismo, al examinar los jueces el peritaje sobre investigación odorológica, de igual manera concluyeron los peritos que las dos huellas olorosas ocupadas de igual manera en el frente de la persiana violentada, obtuvieron coincidencias de olor con el acusado, signos inequívocos de la real responsabilidad de este en los hechos imputado" "2. El tribunal es preciso en valorar el peso que ha tenido en el esclarecimiento de los hechos que las dos huellas olorosas se ocuparan precisamente "en el frente de la persiana violentada"3 y a su vez considera estos indicios como "signos inequívocos de la real responsabilidad de este en los hechos imputados"4.

En otro de los casos estudiados el tribunal en su sentencia argumenta que "Se valoró además de igual manera por el plenario la huella de olor levantada en la superficie del suelo, frente a la maleta violentada, que remitida al Laboratorio Provincial de Criminalística para su examen, se concluyó por los peritos que la misma resultó coincidente con la muestra tomada al acusado, con lo cual se corroboró aún más su participación en este hecho" ${ }^{5}$. En este caso la huella de olor fue levantada frente a la maleta violentada, y este elemento contribuye a conformar la convicción de culpabilidad en el órgano juzgador; no solamente la huella de olor confirma que el acusado estuvo en el lugar de los hechos, sino además cerca del objeto donde se consumó el acto de violencia que constituye un requisito imprescindible para la configuración del delito de Robo con fuerza en las cosas.

Al valorar las pruebas practicadas en juicio oral, practicadas sobre la base de los criterios de la ciencia, la lógica, la razón y la sana crítica, en una causa seguida por la supuesta comisión de un delito de Robo con fuerza en las cosas, se sostiene que entre los elementos tenidos en cuenta por los jueces, para convencerse de la responsabilidad del acusado en los hechos se encontraba "...la ocupación en la inspección al sitio de autos de una huella olorosa, que fue remitida al Laboratorio Provincial de Criminalística para su examen, que acusó corresponderse con la muestra olorosa tomada al reo, lo que corroboró su presencia en el lugar del suceso y su autoría en este hecho que le fue imputado"6 . Nuevamente sirve el dictamen odorológico para "poner" al acusado en el lugar de los hechos, y de soporte a la valoración de los jueces en la búsqueda de la verdad y formación de su certeza de culpabilidad.

2. Sentencia número 74 de 2018 del Tribunal Popular Provincial de Guantánamo.

3. Ídem

4. Ídem

5. Sentencia número 32 de 2019 del Tribunal Popular Provincial de Guantánamo

6. Sentencia número 7 de 2019 del Tribunal Popular Provincial de Guantánamo. 
En otros de los casos estudiados el tribunal juzgador en la valoración de las pruebas, argumenta que "se valoró el acta de la diligencia de reconstrucción de los hechos, protagonizada por ambos acusados; el informe pericial sobre investigación odorológica, en el que se examinó una huella olorosa levantada en el sitio de autos y se concluyó por los peritos que esta resultó coincidente con ambos acusados, todo lo que inexcusablemente los sitúa a ambos en el lugar del hecho"7. Se le dispensa en este caso una importancia vital para demostrar que los acusados estuvieron presentes en el lugar de los hechos, aunque el tribunal valora el resultado del dictamen pericial de odorología en estrecha coincidencia con otras pruebas contenidas en la causa, es especial con la reconstrucción de los hechos.

En su sentencia número 127 el Tribunal Popular Provincial de Guantánamo, argumenta que “... los jueces se convencieron de la responsabilidad del acusado en estos hechos, partiendo precisamente de la confesión que en todo momento del proceso este realizara, lo que no relevó a los juzgadores de la obligación de practicar las pruebas acopiadas, demostrativas de ello... No obvió el Tribunal en su valoración la ocupación de una huella de olor en el sitio de autos que acusó corresponderse con la muestra tomada al acusado, conforme se acreditó mediante el informe de criminalística realizado"8. En este caso queda claro que no basta la confesión del acusado, sino que en todos los casos hay que probar su culpabilidad con independencia de suconfesión o no; para lo cual en el hecho de marra el tribunal se apoyó en el informe pericial criminalístico de odorología.

La huella olorosa contribuye notablemente a demostrar la presencia del imputado en el lugar del hecho, elemento de estimable relevancia en la investigación en la mayoría de los hechos delictivos, y que sirve de prueba para probar su culpabilidad.

En otros de los acusados estudiados, a pesar de la confesión del acusado en la fase de instrucción del proceso penal y en el juicio oral, el órgano juzgador fundamenta en su sentencia que “...también constituyó probanza de este primer hecho el acta de ocupación al acusado de los instrumentos que llevaba consigo y que utilizó para cortar la aldaba de la puerta del bańo afectado y el informe pericial sobre odorología, en el que se estudió la huella olorosa levantada en el lugar del hecho con la muestra ocupada al reo, concluyendo los peritos en cuanto a la coincidencia entre ambas"9 .

En entrevista con los peritos de odorología en la provincia se constató que con frecuencia son citados por el Tribunal correspondiente al juicio oral, regularmente propuestos por la defensa, limitándose está a la confirmación del informe pericial, por lo que el material probatorio obtenido durante la investigación y la instrucción hay que ayudarlo durante el juicio oral para que produzca el efecto, que es el fin, de convicción y certeza del juzgador.

7. Sentencia número 120 de 2019 del Tribunal Popular Provincial de Guantánamo.

8. Sentencia ${ }^{\circ} 127$ de 2017 del Tribunal Popular Provincial de Guantánamo.

9. Sentencia número 116 de 2018 el Tribunal Popular Provincial de Guantánamo. 
Al valorar la importancia del peritaje de odorología criminalística en la fase de instrucción del proceso penal, confirmamos que la valoración del resultado de la investigación de la huella de olor, va alcanzando una mayor jerarquía en la investigación completa, multilateral y objetiva del hecho delictivo.

\section{Seguidamente se exponen algunos ejemplos que confirmar la afirmación anterior:}

- En uno de los casos estudiados al emitir sus conclusiones provisionales, en la investigación de la comisión de un presunto delito de hurto, por un hecho en el cual se había sustraído una embarcación; el fiscal propone como prueba para demostrarla culpabilidad de los acusadosel "Informe pericial sobre investigación odorológica para ilustrar al tribunal las huellas remetidas y los resultados de la primera de la conclusiones en las que acreditan que las huellas de los acusados corresponden con las ocupadas en la embarcación" ${ }^{10}$. El fiscal considera en el caso que las huellas de olor contribuyen a demostrar que los acusados estuvieron en el lugar de los hechos, precisamente dejaron su olor en la embarcación sustraída.

- En el año 2018 al investigar la supuesta comisión de un delito de producción, venta, demanda, tráfico, distribución y tenencia ilícitos de drogas, estupefacientes, sustancias psicotrópicas y otras de efectos similares; una vez concluida la fase de instrucción del proceso, el fiscal sostuvo la acusación entre otras pruebas, en el resultado contenido en el "Informe Pericial sobre investigación odorológica emitido por los peritos ..., los que determinaron que existía coincidencia entre la huella de olor levantada en la embarcación ocupada a los acusados y las impresiones olorosas tomadas a ambos encausados,..." ${ }^{\prime 1}$. Una vez más tiene un efecto esclarecedor la aplicación al caso en cuestión de las posibilidades que aporta la aplicación de la técnica criminalística de odorología.

- Delito de Tráfico de Drogas, una de las pruebas que consideraba practicar en el juicio oral para demostrar la culpabilidad del acusado, el "Dictamen Pericial sobre investigación odorológica..., los que determinaron que existía coincidencia entre la huella de olor levantada en los envoltorios ocupados al acusado y la impresión olorosa tomada al mismo" ${ }^{12}$. Sin duda alguna el acusado tenía que justificar cómo era posible que su huella de olor estuviera impregnada en el envoltorio que contenía la droga. En el caso se lograron acumular otras pruebas, pero sin lugar a dudas la huella de olor fue determinante para confirmar que el acusado tuvo contacto con el envoltorio que contenía la droga.

Es importante considerar la preparación del perito desde la fase de instrucción del proceso penal, para defender el valor probatorio del resultado que se obtenga con la aplicación de la técnica

10. Expediente de Fase Preparatoria N $N^{\circ} 1$ de 2019 de la Unidad Provincial de Investigación Criminal y Operaciones.

11. Expediente de fase preparatoria $N^{\circ} 6$ del 2018 de la División de Investigación Criminal y Operaciones de La Habana.

12. Expediente de fase preparatoria No 19 de 2019 de la Unidad Provincial de Investigación Criminal y Operaciones. 
odorológica. No basta con garantizar la búsqueda, revelación, fijación, extracción, conservación e investigación de la huella de olor, es necesario además en el orden científico ofrecer al instructor, al fiscal y en su momento al tribunal juzgador; la fortaleza de la prueba obtenida, partiendo de argumentar coherentemente su aporte al esclarecimiento del hecho investigado.

Si el especialista no argumenta sólidamente el resultado científico alcanzado, muy poco podrá contribuir a lograr certeza de culpabilidad en el investigador o en los jueces, sobre el valor que se le atribuye a la huella de olor que forme parte del pliego acusatorio.

\section{CONCLUSIONES}

. La especialidad de odorología criminalística es mayoritariamente aceptada, como una prueba de relevancia en la investigación completa, multilateral y objetiva del hecho delictivo.

. En la provincia de Guantánamo es alto el porciento de huellas olor que se extraen del lugar de los hechos en relación al total de huellas levantadas.

. Se comprobó la existencia de una alta efectividad en la búsqueda, revelación, fijación, extracción, conservación e investigación de la huella de olor.

. Los tribunales cubanos ponderan el resultado del dictamen pericial de la huella de olor, como parte de las valoraciones que realizan para llegar a la convicción y certeza plena de culpabilidad del acusado en el hecho delictivo que se le imputa.

. A pesar de su probada utilidad en la investigación criminal, aún no es suficientemente empleado el dictamen pericial de huella de olor, en el esclarecimiento del hecho presuntamente delictivo.

\section{REFERENCIAS}

- Colectivo de autores: Criminalística. La Habana: Editorial Universitaria Félix Varela. 2015.

- Colectivo de autores: Temas de Criminalistica. La Habana: Editorial Universitaria Félix Varela. 2007.

- Hernández de la Torre, Rafael. La criminalistica en preguntas y respuestas. Colección Jurídica. La Habana: Editorial de Ciencias Sociales, (2016). Impreso en la UEB Gráfica de Holguín.

- $\quad$ Expediente de fase preparatoria N 6 del 2018 de la División de Investigación Criminal y Operaciones de La Habana. https://doi.org/10.29057/icea.v6i11.2709 
- $\quad$ Expediente de fase preparatoria No. 19 de 2019 de la Unidad Provincial de Investigación Criminal y Operaciones.

- Expediente de Fase Preparatoria No. 1 de 2019 de la Unidad Provincial de Investigación Criminal y Operaciones.

- Sentencia Nº127 de 2017 del Tribunal Popular Provincial de Guantánamo.

- $\quad$ Sentencia N 74 de 2018 del Tribunal Popular Provincial de Guantánamo.

- Sentencia N 116 de 2018 del Tribunal Popular Provincial de Guantánamo.

- Sentencia Nº 7 de 2019 del Tribunal Popular Provincial de Guantánamo.

- Sentencia N 32 de 2019 del Tribunal Popular Provincial de Guantánamo.

- Sentencia N 120 de 2019 del Tribunal Popular Provincial de Guantánamo.

RECIBIDO: $22 / 02 / 20$

APROBADO: $20 / 05 / 20$ 


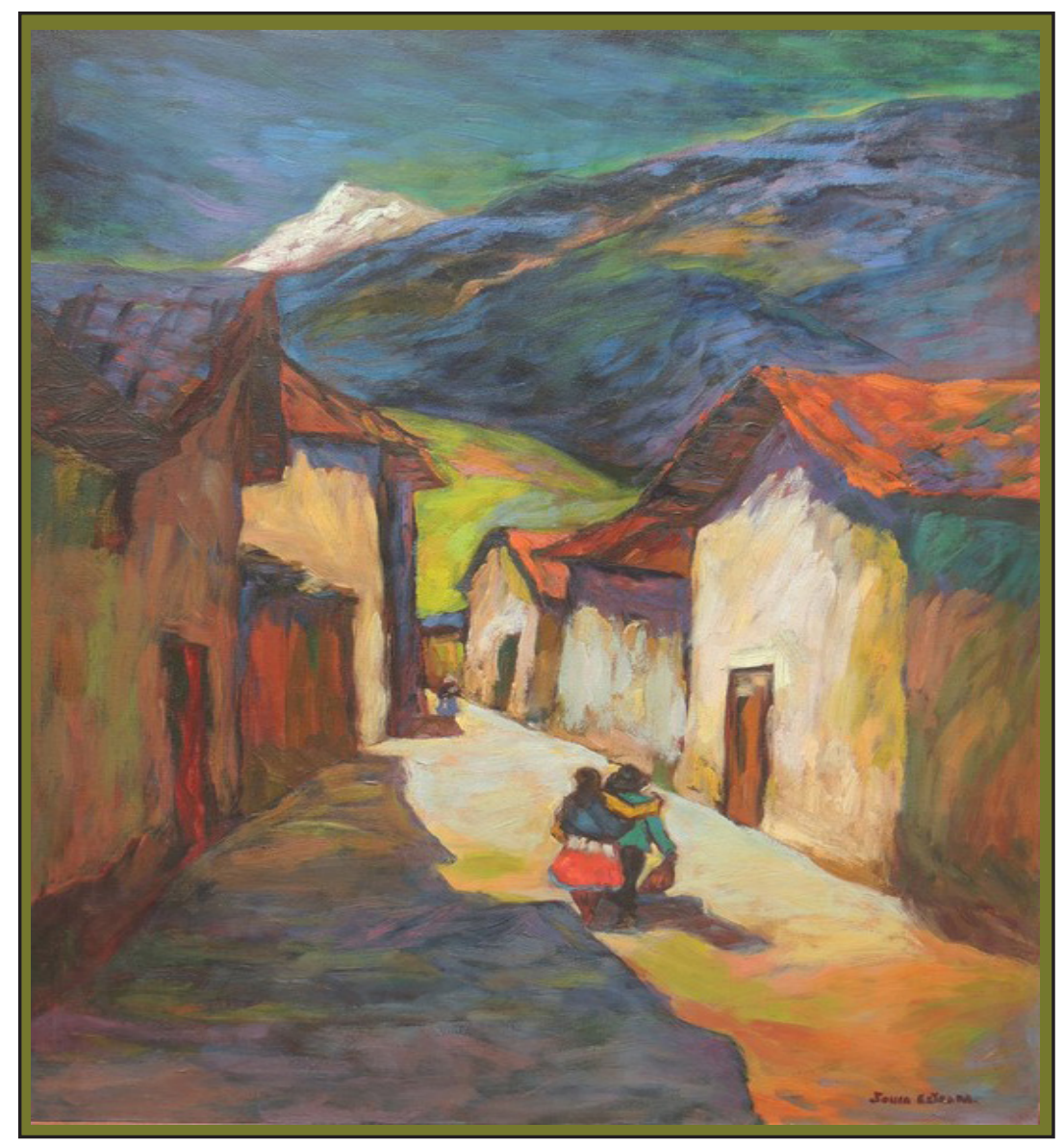

El Champará. Óleo sobre lienzo 100 x $81 \mathrm{~cm}$. Sonia Estrada Melgarejo (pintora peruana, Ancash 